\title{
The Management System of Remote Equipment Base on SMS
}

\author{
Wei Yan \\ College of Physics and Electronic Information, Inner Mongolia University for the Nationalities, \\ Tongliao, Inner Mongolia 028000, China
}

Keyword: GMS, SMS, single chip computus, AT instructions, long-distance control

\begin{abstract}
With the unceasing development of national economy and science and technology, wireless data transmission is extensive used in many areas, basing on its characteristic of flexible and so on. Based on the SMS of GSM net, this article introduce an intelligentized surveillance system which is used for controlling the long-distance leakage protector. The current GSM system connects wireless data transmission system and the traditional leakage protector, and the control system detects leakage protector and communicates of the data, making the system more intelligent and practical.
\end{abstract}

\section{Introduction}

In recent years, the GSM digital mobile communication network with the rapid development of construction, is currently the most extensive coverage, system reliability, the highest number of mobile cellular communication systems, with strong function of authentication and encryption, the system anti-interference ability, reliable system operation ${ }^{[1]}$. With the continuous expansion of GSM network applications, the business was more and more open types, including the short message service (SMS) which have a wide range of industrial control applications, commonly used in long-distance controling and locale data transmission.

This paper introduces a kind of intelligent remote management system based on short message service in GSM network and cloud manage more than one leakage protection ${ }^{[2]}$. GSM network as a basic service, SMS for its simple, low cost communications, high spectrum efficiency, system capacity, many types of business, anti-interference ability, international automatic roaming features, in many fields has been widely used, for example remote maintenance, data acquisition systems, etc..

\section{System structure and working principle}

\subsection{Structure of the system}

The leakage protection remote management system which based on short message function of GSM mobile communications system, mainly consists of the central controlled module, the short messaging module, timing module, the keyboard input module and information display module. System block diagram is shown in Figure 1:

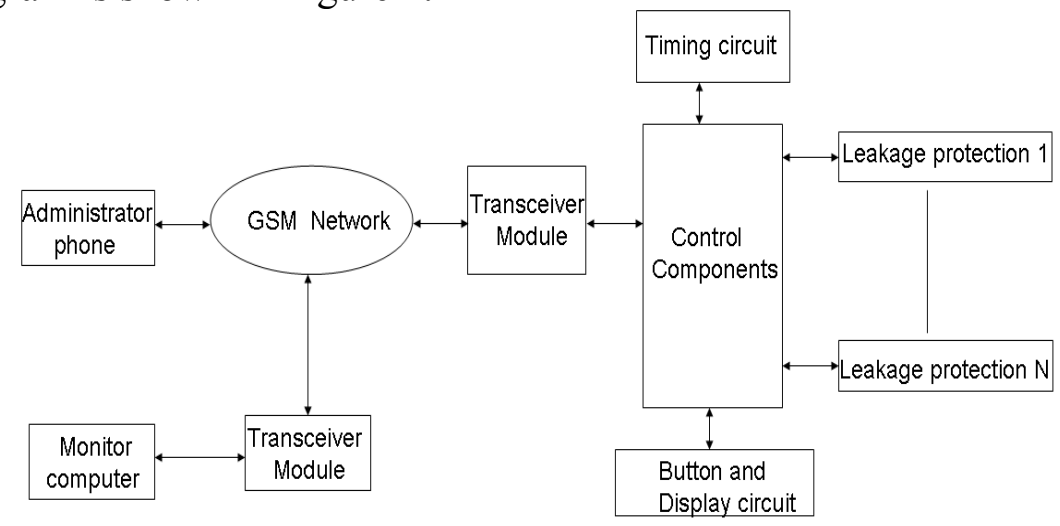

Figure 1 System diagram 


\subsection{System works}

The center control module of the system connected to various leakage protection in low-voltage AC current, when any line due to overload, short circuit, overvoltage, undervoltage or residual current trips would send an alarm message.Via SMS transceiver module send a short message to managemer's phone and monitor computer, which includes alarm place, alarm line, alarm reason and the trouble time,etc. Managers can quickly troubleshoot based on SMS to ensure reliability and continuity of power supply. At the same time, managers can collect the data of each line by sending a short message to make sure the management of fixed-line monitoring.

\section{System hardware Circuit Design}

The system hardware circuit uses AT89C51 microcontroller as a central control module, and makes external expansion decoder connect to many leakage protection device to save I / O port resources; Short messaging module using the higher cost-effective TC35i chip make by Siemens, which sets RF and baseband in together and provide a standard AT command interface to users for fast, reliable, secure transmission of data, voice, SMS and fax.Users can use TC35i develop and design system conveniently ${ }^{[4]}$. TC35i and GSM2/2+ are compatible, and it own dual-band (GSM900/GSM1800), RS232 data port, in line with ETSI standard GSM07.07 and GSM07.O5; Display module with LCD display shows the administrator's phone number.Users can set and change the phone number through the system keyboard.

3.1 Main hardware circuits

\section{(1) TC35i interface circuit ${ }^{[5]}$}

At present, GSM module kind mainly including the 2D series of CENTEL PIML, the TC35 series of Siemens, the WMO2 series of Wavecome, Ericsson DM10/DM20 series, ZTE ZXGM18 series and so on,but these function and usage of the module are not must different.Siemens TC35 series modules with higher cost-effective has a domestic radio network cards. So the design choose the TC35i of Siemens TC35 series as the module.This is the new wireless modules of Siemens, functionally compatible with the TC35,compact designed,that greatly reduces the volume of products. TC35i chip has 40 ports, and 1 to 14 pins for power supply; 24 29 pins for SIM card, respectively as CCIN, CCRST, CCIO, CCCLK, CCVCC and CCGND. 33 to 40 pins for the voice interface, 15 pin is the IGT (Ignition) that connects the P3.4 of MCU, so as to start the TC35 chip by a pulse.The port TXD through a buffer links the 18-pin of TC35i, and RXD through the buffer links the 19-pin of TC35i. The circuit of TC35i module showns in Figure 2.1:

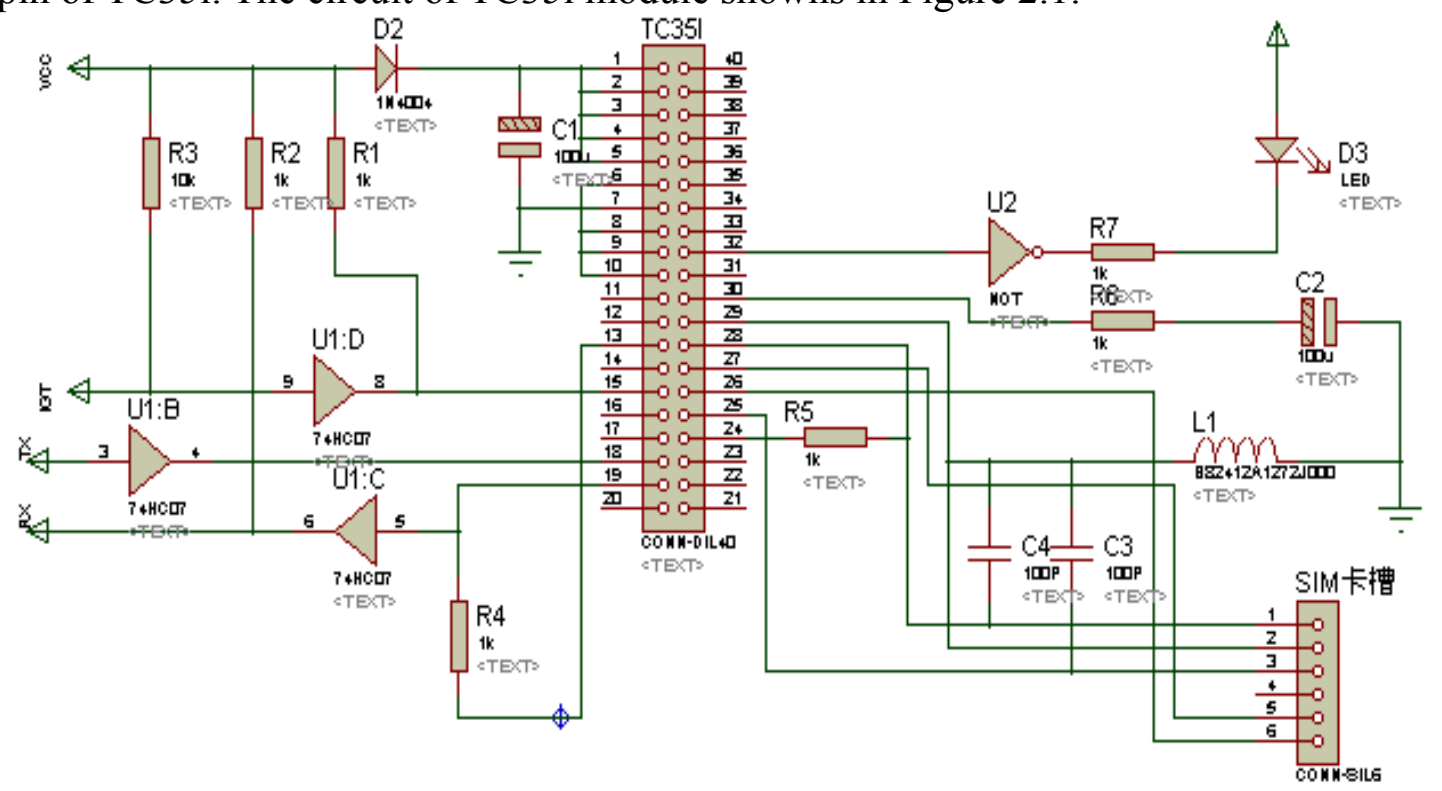

Figure 2.1 The circuit of TC35i module 
(2) Time circuit

Since leakage protection system needs to record the specific time of leaker protection action, make the introduction of timing circuits. The DS1307 timing chip of DALLAS as a $\mathrm{I}^{2} \mathrm{C}$ bus interface clock calendar chip, using two lines to communicate with the CPU, with counting function of seconds, minutes, hours, days, weeks, months and years, which has 12-hour and 24-hour counting mode, automatically adjusts the number of days per month and leap year, and also has an automatic power-down protection and reset function ${ }^{[6]}$. Considering the simplicity and cost-effective of system operation, it is necessary to use DS1307 chip as the core of the system timing circuit. The external connected circuit of DS1307 is shown in Figure 2.3:

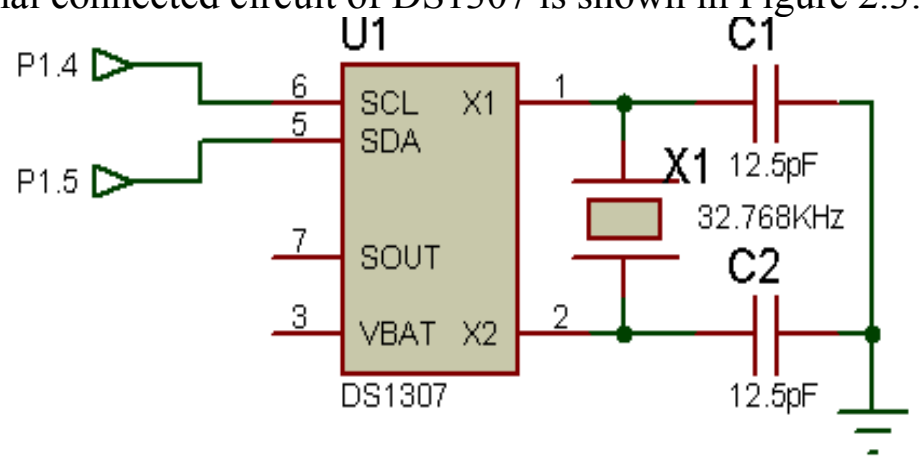

Figure 2. 3 The external connected circuit of DS1307

\section{System Software Design}

The main functions of System softwar are: (1) connecting to real-time monitoring of leakage protection, analysising and processing the data transmissed;(2)In accordance with a coding mode, making the bidirectional communication with the controllor through the GSM module;(3) carring out the keyboard and display that can change the administrator phone number through the keys, and information displayed on the LCD unit.System Control GSM module through the AT command.The short message encoding uses the PDU encoding mode that can send characters in Chinese and English and widely used.

\subsection{Short message encoding and AT command set}

There are three modes of short message encoding: Block Mode, Text Mode, PDU Mode. PDU mode in which is the most widely used.PDU mode as a way is sending or receiving short messages, the short message text can be sent out after the hexadecimal codes.PDU Mode supported by all phones can use any character set, which is also the default short message encoding ${ }^{[7]}$.

AT (Attention) command set is the industry standard of modem's communication interface released by the ETSI (European Telecommunications Technology Committee), which contains instructions for the control of SMS. PC sends AT commands via serial port of the TC35i module, through the serial port reading and writing operations in order to achieve function on sending and receiving.

\subsection{System Software and the main program flow chart}

When sending trouble alarm message software process: MCU goes on real-time monitoring to the leakage protection connected, once the circuit gets a malfunction, leakage protection acts and the MCU analyze the data and sent an alarm short message to the management by the GSM module, when the management received short message successfully, the system automatically returns "OK" and prompt short message sent successfully, if the short message sending fails, the system re-issues information after a short delay to ensure the success of receiving failure alarm information. Sending alarm information flow is shown in Figure 3.1: 


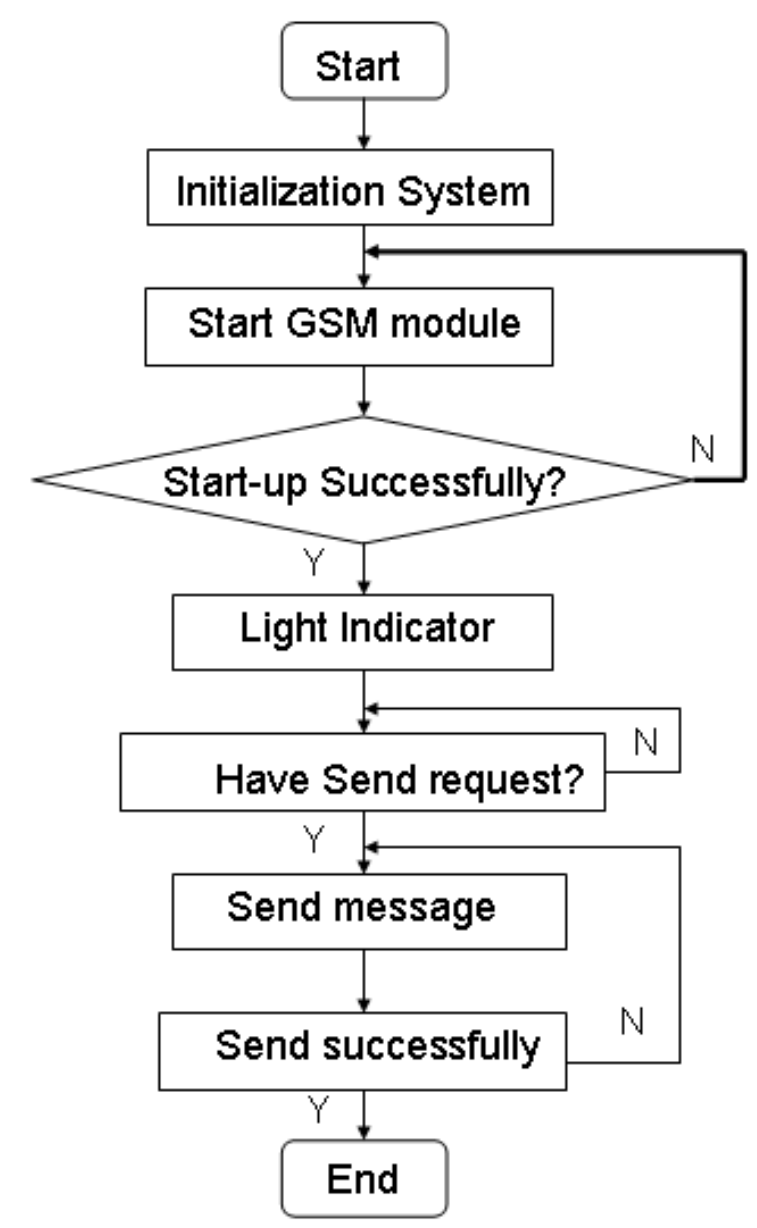

Figure 3.1 Information flow failure alarm sent

\section{Conclusion}

Make the MCU as the control core and apply Short Message Service in GSM network, to achieve remote monitoring and controlling by SMS.Make the system application more flexible and save a lot of manpower, material resources, and to achieve protection, telemetry, communications, remote controlling in one.

\section{References}

[1] Xie Chuan Luan, Xing Enfu. Based on short message leakage protection remote management system. Manufacture of industrial automation, 2009 (1) :123-125

[2] Chen Zhen Ping, Liu Xia Huai. Thin Circuit Breaker Controller. Coal Mine Machinery, 2004 (9) :29-30

[3] Hou Dawei, He Jianzhong GSM-based Remote Data Acquisition and Control System Computer Engineering and Design 2009 (9): 30

[4] Li Zhiqiang, HONGKONG granary GSM-based wireless monitoring system temperature and humidity of Agricultural Mechanization Research 2009 (9) :88-91

[5] Weiying Qin Chen Liankun TC35i's PC-based messaging module electronic world, 2008 (6)

[6] Guan Jian Wang. "User-programmed clock control circuits." Electronic world. 1999, (2) ,21-22.

[7] Chi-Hsiang Wu, Rong-Hong Jan. System integration of WAP and SMS for home network system. Computer Networks， 2003 (7): 493 502 